\title{
Phenomenological analysis of a quark mass texture
}

\author{
Andrea Romanino* \\ Department of Physics, Theoretical Physics, University of Oxford, Oxford OX1 3NP, UK \\ E-mail: 'romanino@thphys ox. ac.uki,
}

ABstract: The relations $\left|V_{u b} / V_{c b}\right|=\left(m_{u} / m_{c}\right)^{1 / 2}$ and $\left|V_{t d} / V_{t s}\right|=\left(m_{d} / m_{s}\right)^{1 / 2}$ between the CKM matrix elements and the quark masses are shown to imply a remarkably precise determination of the CKM unitarity triangle or of the Wolfenstein parameters $\rho, \eta$ consistent with the data so far. We view this as a clean test of a quark mass texture neatly arising from a hierarchical breaking of a flavour symmetry.

\section{Introduction}

While the structure of the lepton flavour interactions in the Standard Model (SM) is going to be generalized to take neutrino masses and mixings into account, the description of the quark flavour interactions given in the SM in terms of 10 physical parameters, 6 masses, 3 angles and one CPviolating phase is, from a phenomenological point of view, very well established. The 6 masses and 2 out of the 4 mixing parameters, $\left|V_{u s}\right|$ and $\left|V_{c b}\right|$, are very well measured, and a rather good direct measurement of the third mixing parameter, $\left|V_{u b} / V_{c b}\right|$, is also available. Moreover, the quark flavour physics is well accounted for in terms of this description.

On the other hand, from the theoretical point of view, the situation is much less clear. Many theories have been proposed to explain the pattern of masses and mixings based especially on the spontaneous breaking of flavour symmetries of different kind, discrete or continuous, abelian or non-abelian, but all in qualitative agreement with the available data. This raises the question whether it is possible to go beyond the qualitative agreement shared by all models and to test experimentally not only the phenomenology of the flavour sector but also the underlying theory giving rise to it. This is the issue that will be

\footnotetext{
*This work was supported in part by the TMR Network under the EEC Contract No. ERBFMRXCT960090.
}

addressed here.

\section{The texture}

In a flavour theory, the quark masses and mixings are obtained by diagonalising the mass matrices for the up and down quarks ${ }^{1}$

$$
M^{U, D}=\left(\begin{array}{lll}
M_{11} & M_{12} & M_{13} \\
M_{21} & M_{22} & M_{23} \\
M_{31} & M_{32} & M_{33}
\end{array}\right)^{U, D}
$$

obtained in terms of the breaking of a flavour symmetry. We will not describe here the details of how the mass matrices are obtained. It is not surprising however that in most cases the breaking of the symmetry makes small parameters available, so that hierarchical textures for the mass matrices are obtained, the hierarchy corresponding to the hierarchy of the quark masses,

$$
\left|M_{33}\right| \sim m_{3} \quad\left|\begin{array}{ll}
M_{22} & M_{23} \\
M_{32} & M_{33}
\end{array}\right| \sim m_{2} m_{3}
$$

both in the $U$ and $D$ sector ${ }^{2}$.

Among the possible hierarchical textures, one of the most popular in the literature is the $1-2$ symmetric "texture-zero", defined by

$$
M_{11}=M_{13}=M_{31}=0, \quad\left|M_{12}\right|=\left|M_{21}\right|,
$$

\footnotetext{
${ }^{1}$ In the following formula the right indexes correspond to the right-handed fermions and the left indexes to the left-handed ones.

${ }^{2}$ The third relation $|\operatorname{det} M|=m_{1} m_{2} m_{3}$ does not depend of course on the hierarchical hypothesis.
} 
that has the virtue of producing the following predictions for CKM matrix elements in terms

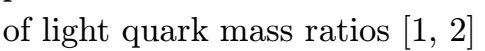

$$
\left|V_{u s}\right|=\left|\sqrt{\frac{m_{d}}{m_{s}}}+e^{i \phi} \sqrt{\frac{m_{u}}{m_{c}}}\right|
$$

and [i]']

$$
\left|\frac{V_{u b}}{V_{c b}}\right|=\sqrt{\frac{m_{u}}{m_{c}}} \quad\left|\frac{V_{t d}}{V_{t s}}\right|=\sqrt{\frac{m_{d}}{m_{s}}},
$$

up to corrections discussed below, that are quite in a good agreement with the experimental values. By the way, the texture $(2.3)$ is the only one that can generate $(2 . \overline{4})$ and $(2.51)$ in a non accidental way [2].

In the following, we will show that a quantitative analysis of the simple relations $(2,5)$, can provide a precise test of the texture (2.3) giving rise to them and in turn of the flavour model producing the texture. Among such models, we quote here theories based on continuous abelian $\mathrm{U}(1)$ flavour groups [4i] $M_{12} \simeq M_{21}$ has to be given as an additional hypothesis, and non-abelian $\mathrm{U}(2)$ theories [ip], in which $M_{12} \simeq M_{21}$ is related to the symmetry properties of the irreducible representations of the non-abelian group.

\section{Corrections and model-dependen- ce}

Before skipping directly to the results we shortly discuss the corrections to the relations (2.5).

The overall corrections to the two relations in $\left(\begin{array}{c}2 . \overline{3} \\ -1\end{array}\right)$ turn out to be equal in absolute value, meaning that the relative importance of the correction is larger for the $\left|V_{u b} / V_{c b}\right|$ relation than for the $\left|V_{t d} / V_{t s}\right|$ one. In the following analysis, possible corrections up to $10 \%$ in the $\left|V_{u b} / V_{c b}\right|$ relation will be taken into account.

There are two sources of corrections to the relations $(2.4,2.5)$, both depending on the details of the model giving rise as a first approximation to the texture $(2.3 i)$. The first source is due to the fact that in real situations the relations (2.3i) are not exact. The amount of correction depends on the model. A $10 \%$ correction to the $\left|V_{u b} / V_{c b}\right|$ relation takes into account non-vanishing $M_{13,31}^{U, D}$ with

$$
\left|M_{13,31}^{U}\right| \lesssim 200 \% m_{u} \quad\left|M_{13,31}^{D}\right| \lesssim 20 \% m_{d}
$$

and an asymmetry between $\left|M_{12}^{U, D}\right|$ and $\left|M_{21}^{U, D}\right|$ with

$$
|| \frac{M_{12}^{U}}{M_{21}^{U}}|-1| \lesssim 20 \%
$$

Even in the ideal case in which eqs. (2.2.3) are exact, corrections to the angle-mass relations (2. come from diagonalisation of the " $2-3$ " block of the mass matrices. The diagonalisation of $M^{U, D}$ can in fact be performed through three subsequent rotations in the $2-3,1-2$ and $1-3$ sectors. Due to the hierarchical structure (2.2i), the left and right rotation diagonalising the $2-3$ sector have to be performed first. But the $2-3$ right rotation of angle $\theta_{23}^{R}$ also acts on the $M_{12}$ matrix element rotating it a bit in the $M_{13}$ one. Therefore a $M_{13} \sim M_{12} \tan \theta_{23}^{R}$ entry is generated, giving a contribution to the 1-3 left rotation that spoils eqs. $(2 \cdot 2)$. This correction depends on the model too through the structure of the 2-3 sector. However, barring cancellations or large $M_{23}-M_{32}$ asymmetries, this correction does not affect the $\left|V_{u b} / V_{c b}\right|$ relation more than $10 \%$.

\section{Analysis}

The relations (2.5) allow a determination of the less known CKM parameters in terms of light quark masses. The obvious way of testing their validity is therefore to compare this prediction with the experimental determination of the CKM parameters. Less obvious is that the prediction in terms of quark masses is precise enough to constitute a good test. This is in fact the case, as we will see in a moment. In the following we will use the Wolfenstein parameterisation $\left[\overline{\sigma_{1}}\right]$ of the CKM matrix in terms of $\lambda, A, \bar{\rho}, \bar{\eta}$, where $\bar{\rho}, \bar{\eta}$ are connected to the original $\rho, \eta$ parameters by $\bar{\rho}=c \rho, \bar{\eta}=c \eta$, where $c=\sqrt{1-\lambda^{2}}$. In terms of these parameters and using the phase convention of the standard parameterisation,

$$
\begin{array}{ll}
V_{u s}=\lambda & V_{u b}=A \lambda^{3}(\rho-i \eta) \\
V_{c b}=A \lambda^{2} & V_{t d}=A \lambda^{3}(1-\bar{\rho}-i \bar{\eta}) .
\end{array}
$$


In fig. 'ili, the prediction for $(\bar{\rho}, \bar{\eta})$ (smaller regions) is compared with the present indirect experimental determination through a SM fit (larger regions). The inputs for this last determination include the direct measurement of $\left|V_{u b} / V_{c b}\right|$ and the indirect information from the mixing in the $B_{d}$-system, $\Delta m_{B_{d}}$, and in the $B_{s}$ system, $\Delta m_{B_{s}}$. We prefer not to include in the SM fit the parameter $\epsilon_{K}$ since we leave open the possibility that some extra sources of CP-violation not included in the SM may exist, affecting in particular CPviolation in the Kaon system. As a consequence, the sign of $\bar{\eta}$ is not determined so that figure represent only the $\bar{\eta}>0$ part of a figure symmetric with respect to the $\bar{\eta}$ axes. However, the recent measurement of $\sin 2 \beta$, if included in the fit, would just cut out the not showed part of the distribution. The inclusion of $\epsilon_{K}$ in the SM fit would not alter the agreement between the predicted $\bar{\rho}, \bar{\eta}$ and present data. To see this, the constraint given by $\epsilon_{K}$ is shown independently in fig. possibility that physics beyond the SM give a contribution to $\epsilon_{K}$ equal to $0,1 / 3,2 / 3$ of the measured value. The light-shadowed region is excluded by eqs. (2.5i).

From figure ${ }_{1}^{1}$ we see that the prediction is presently in quite a good agreement with the experimental determination (the $1 \sigma$ shift being mainly due to the lower value of the predicted $\left|V_{u b} / V_{c b}\right|$ compared to the measured one). Moreover we see that the prediction in terms of light quark masses is tight enough to represent a very good test of the texture giving rise to it. $B$ factories will give either a strong evidence or will rule out this texture and the flavour models generating it.

To understand the tightness of the prediction, as well as the reason of the exclusion of a large part of the $\bar{\rho}-\bar{\eta}$ plane, we will describe now in better detail the procedure used to implement eqs. $(2.51)$. The simplest implementation would consist in fitting the experimental values of $m_{u} / m_{c}$ and $m_{d} / m_{s}$ in terms of $\bar{\rho}$ and $\bar{\eta}$ using (2.5). On the other hand, the lightest quarks $m_{u}$ and $m_{d}$ are better known through the ratios $m_{u} / m_{d}$ and $m_{d} / m_{s}$, so that $m_{u} / m_{c}$ and $m_{d} / m_{s}$ have to be recovered in terms of the values of $m_{u} / m_{d}, m_{d} / m_{s}$ and $m_{c} / m_{s}$. In turn, $m_{u} / m_{d}$ and $m_{d} / m_{s}$ can be conveniently expressed in terms of $m_{u} / m_{d}$ and $Q$, where $Q$ is defined by

$$
Q=\frac{m_{s} / m_{d}}{\sqrt{1-\left(m_{u} / m_{d}\right)^{2}}} .
$$

$Q$ is in fact known much more precisely than $m_{u} / m_{d}$ and $m_{d} / m_{s}$, both in the sense that its error is smaller and that its determination is relatively more reliable than the $m_{u} / m_{d}$ or $m_{d} / m_{s}$ one, since it is based only on second order chiral perturbation theory i7. For the purpose of the present discussion we can consider $Q$ as fixed and consider the smaller region in fig. termined by the two constraints obtained by expressing $m_{u} / m_{d}$ and $m_{c} / m_{s}$ in terms of $\bar{\rho}$ and $\bar{\eta}$. The two constraints are plotted separately in fig. 1'r. The $m_{u} / m_{d}$ constraint is represented by the dashed circumferences around $(1,0) . m_{u} / m_{d}$ is in fact directly related to the radius of such circumferences, since

$$
\begin{aligned}
& \frac{\lambda}{c} \sqrt{(1-\bar{\rho})^{2}+\bar{\eta}^{2}}=\left|\frac{V_{t d}}{V_{t s}}\right| \\
& =\sqrt{\frac{m_{d}}{m_{s}}}=\left(\frac{1}{Q \sqrt{1-\left(m_{u} / m_{d}\right)^{2}}}\right)^{1 / 2},
\end{aligned}
$$

that also shows there is a minimum value of the radius, corresponding to $m_{u} / m_{d}=0$ (excluded region). The $m_{c} / m_{s}$ constraint is represented instead by the two approximate half-circumferences around the origin meeting on the positive $\bar{\rho}$ axes. We can now see why the prediction is so tight. On one hand, the first constraint is not very sensitive to the error on $m_{u} / m_{d}$, since the dependence on $m_{u} / m_{d}$ only comes through $m_{d} / m_{s}$ and is therefore relatively small (see eq. $\left(\begin{array}{l}A \\ -\end{array} \overline{\overline{3}} \overline{1}\right)$ )). On the other hand the dependence of the second constraint on the error of $m_{c} / m_{s}$ and on the incertitudes of the $\left|V_{u b} / V_{c b}\right|$ relation is small where the two constraints intersect due to the closeness of the exclusion region (this dependence is three times smaller than on the opposite side). The input values for the parameters involved in both

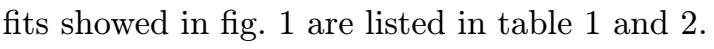

\section{Potential improvements of the com- parison with data}

The prospects for an improved comparison of the 


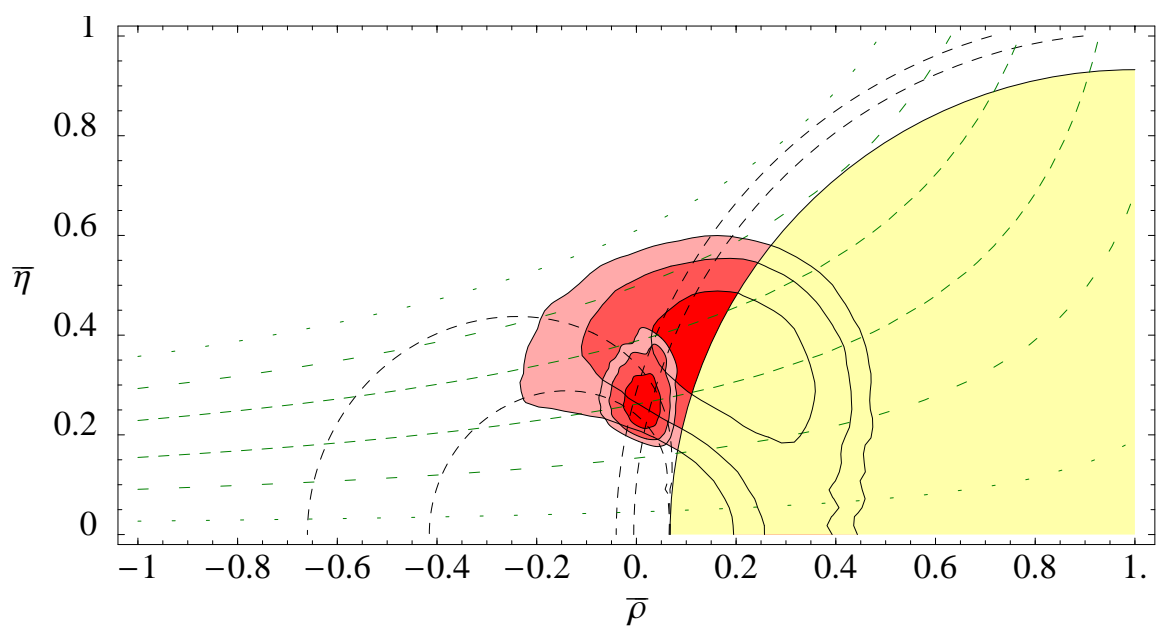

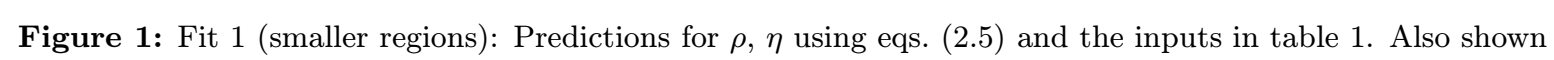
are the two individual constraints from $m_{u} / m_{d}$ and $m_{c} / m_{s}$ separately (see text). Fit 2 (larger regions): SM fit using $\left|V_{u b} / V_{c b}\right|, \Delta m_{B_{d}}, \Delta m_{B_{s}}$ but not $\epsilon_{K}$, whose constraint is shown independently with different theoretical errors (see text). For both fits the contours are at 68, 95 and $99 \%$ CL respectively.

\begin{tabular}{|c|c|}
\hline$Q$ & $22.7 \pm 0.8$ \\
$m_{u} / m_{d}$ & $0.553 \pm 0.043$ \\
$m_{c} / m_{s}$ & $8.23 \pm 1.5$ \\
\hline
\end{tabular}

Table 1: Input values for the quark mass ratios. $Q$ is defined in the text.

predictions of the texture $\left(22^{-} \cdot \overline{3}\right)$ with further data are clear from fig ${ }_{1}^{1} \overline{1}$. Another way to illustrate this is in fig ${ }_{2}^{12}$ Shown are the probability distributions for $\bar{\rho}, \bar{\eta}$ and for various physical quantities obtained by a combined fit of $\left|V_{u b} / V_{c b}\right|$, $\Delta m_{B_{d}}, \Delta m_{B_{s}}$ and the constraints (2.5.j). These distributions are compared with those from a pure SM fit with present data and $\epsilon_{K}$ still not included, but rather shown as an output (fig $\left.\overline{2}_{2} \mathrm{~d}\right)^{3}$. Based on this figure one can be optimistic on the possibility of a stringent comparison with data to come.

It is less clear, in fact, that an improvement may come from an independent better determination of the inputs in table 11.' On the contrary, one has to keep in mind that the theoretical de-

\footnotetext{
${ }^{3}$ The same distributions with the inclusion of $\epsilon_{K}$ remain essentially unchanged except for cutting away the low $\eta$-region and consequently shifting $\sin 2 \alpha$ more toward zero.
}

termination of $m_{u} / m_{d}$ is not on the same ground as for the two other parameters. For this reason the prediction for various physical observables is shown in fig $\overline{1}$ as function of $m_{u} / m_{d}$ and $\eta>0$. Since some of these observables have a significant dependence on this ratio, with better data it would be useful to leave even $m_{u} / m_{d}$ as a free parameter. Notice in fig 21 the preferred value of $\left|V_{u b} / V_{c b}\right|$ relative to the present determination, mostly affected by theoretical uncertainties. Notice also in figs on $\Delta m_{B_{s}}$.

\section{Conclusions}

The relations $\left|V_{u b} / V_{c b}\right|=\left(m_{u} / m_{c}\right)^{1 / 2}$ and $\left|V_{t d} / V_{t s}\right|=$ $\left(m_{d} / m_{s}\right)^{1 / 2}$ arise in any scheme of hierarchical quark masses where the 11, 13 and 31 entries are sufficiently small, and the 12 and 21 entries are equal up to a phase. Using the Wolfenstein form for the CKM matrix, we have shown that these two relations can be translated into a tight prediction in the $\bar{\rho}-\bar{\eta}$ plane. The predicted region is significantly smaller than that currently allowed by data, as shown in figure $\overline{1}_{\mathrm{r}}^{\mathrm{r}}$ the texture successfully accounts for the present data and will be subject to further stringent tests by future data.

The most important implications for future experiments are: a deviation from complete $B_{s}$ 


\begin{tabular}{||c|c||c|c||}
\hline$G_{F}$ & $1.16639 \cdot 10^{-5} \mathrm{GeV}^{-2}$ & $M_{W}$ & 80.375 \\
$\lambda$ & 0.2196 & $A$ & $0.819 \pm 0.035$ \\
$m_{B_{d}}$ & $(5.2792 \pm 0.0018) \mathrm{GeV}$ & $m_{B_{s}}$ & $(5.3692 \pm 0.0020) \mathrm{GeV}$ \\
$f_{B_{d}} \sqrt{B_{B_{d}}}$ & $(0.201 \pm 0.042) \mathrm{GeV}$ & $\zeta$ & $1.14 \pm 0.08$ \\
$\eta_{B}$ & $0.55 \pm 0.01$ & $m_{t}$ & $(166.8 \pm 5.3) \mathrm{GeV}$ \\
$\Delta m_{B_{d}}$ & $(0.471 \pm 0.016) \mathrm{ps}^{-1}$ & $\epsilon_{K}$ & $(2.280 \pm 0.019) \cdot 10^{-3}$ \\
$B_{K}$ & $0.87 \pm 0.14$ & $f_{K}$ & $(0.1598 \pm 0.0015) \mathrm{GeV}$ \\
$m_{K}$ & $(0.497672 \pm 0.000031) \mathrm{GeV}$ & $\Delta m_{K}$ & $(3.491 \pm 0.009) \cdot 10^{-12} \mathrm{MeV}$ \\
$\eta_{1}$ & $1.38 \pm 0.53$ & $\eta_{2}$ & $0.574 \pm 0.004$ \\
$\eta_{3}$ & $0.47 \pm 0.04$ & $\left|V_{u b} / V_{c b}\right|$ & $0.093 \pm 0.016$ \\
\hline
\end{tabular}

Table 2: Inputs for the SM fit.

mixing must be discovered soon, $\Delta m_{B_{s}}<14.9 \mathrm{ps}^{-1}$ at $90 \%$ c.l., and the predicted probability distributions for $|\sin 2 \alpha|$ and $|\sin 2 \beta|$ are both peaked near 0.5 , as shown in figures $2, a, b$,e. These results use $m_{u} / m_{d}=0.55 \pm 0.04$ as an input. However, even if this input is completely relaxed, so that only one combination of the two light mass ratios is used, the upper bound on $B_{s}$ mixing remains robust: $\Delta m_{B_{s}}<15 \mathrm{ps}^{-1}$ at $90 \%$ c.l. In this case significant variations in $\sin 2 \alpha$ and $\sin 2 \beta$ are possible, as shown in figures $\overline{3}_{1} \mathrm{a}, \mathrm{b}$. Note in any case that lower values of $m_{u} / m_{d}$, that could accommodate a higher $\Delta m_{B_{s}}$, push further down the expected value of $\left|V_{u b} / V_{c b}\right|$, in apparent contradiction with the present determination.

These results are all independent of whether there is a significant non-standard model contribution to $\epsilon_{K}$. However, the size of such an exotic contribution is restricted by the form of the texture, as shown in figure inf. In the absence of such a contribution, the combination of figures and 3 predicts that $m_{u} / m_{d}$ is not far from the commonly accepted value of $0.55 \pm 0.04$.

\section{References}

[1] H. Fritzsch, Phys. Lett. 73B (1978) 317.

M. Shin, Phys. Lett. 145B (1984) 285.

M. Gronau, R. Johnson, and J. Schechter, Phys. Rev. Lett. 54 (1985) 2176.

[2] L. J. Hall and A. Rasin, Phys. Lett. B315 (1993) 164.

[3] H. Fritzsch, Nucl. Phys. B155 (1979) 189.
B. Stech, Phys. Lett. 130B (1983) 189.

[4] L. Ibanez and G. G. Ross, $p l$ B332 (1994) 100-110, hepeph/9403338i.

[5] R. Barbieri, G. Dvali, and L. J. Hall, Phys. Lett. B377 (1996) 76.

R. Barbieri, L. J. Hall, S. Raby, and

A. Romanino, Nucl. Phys. B493 (1997) 3.

R. Barbieri, L. J. Hall, and A. Romanino, Phys. Lett. B401 (1997) 47.

[6] L. Wolfenstein, Phys. Rev. Lett. 51 (1983) 1945.

[7] H. Leutwyler, "The masses of the light quarks." Talk given at the Conference on Fundamental Interactions of Elementary Particles, ITEP, Moscow, Russia, Oct., 1995. hep-ph/9602255. 

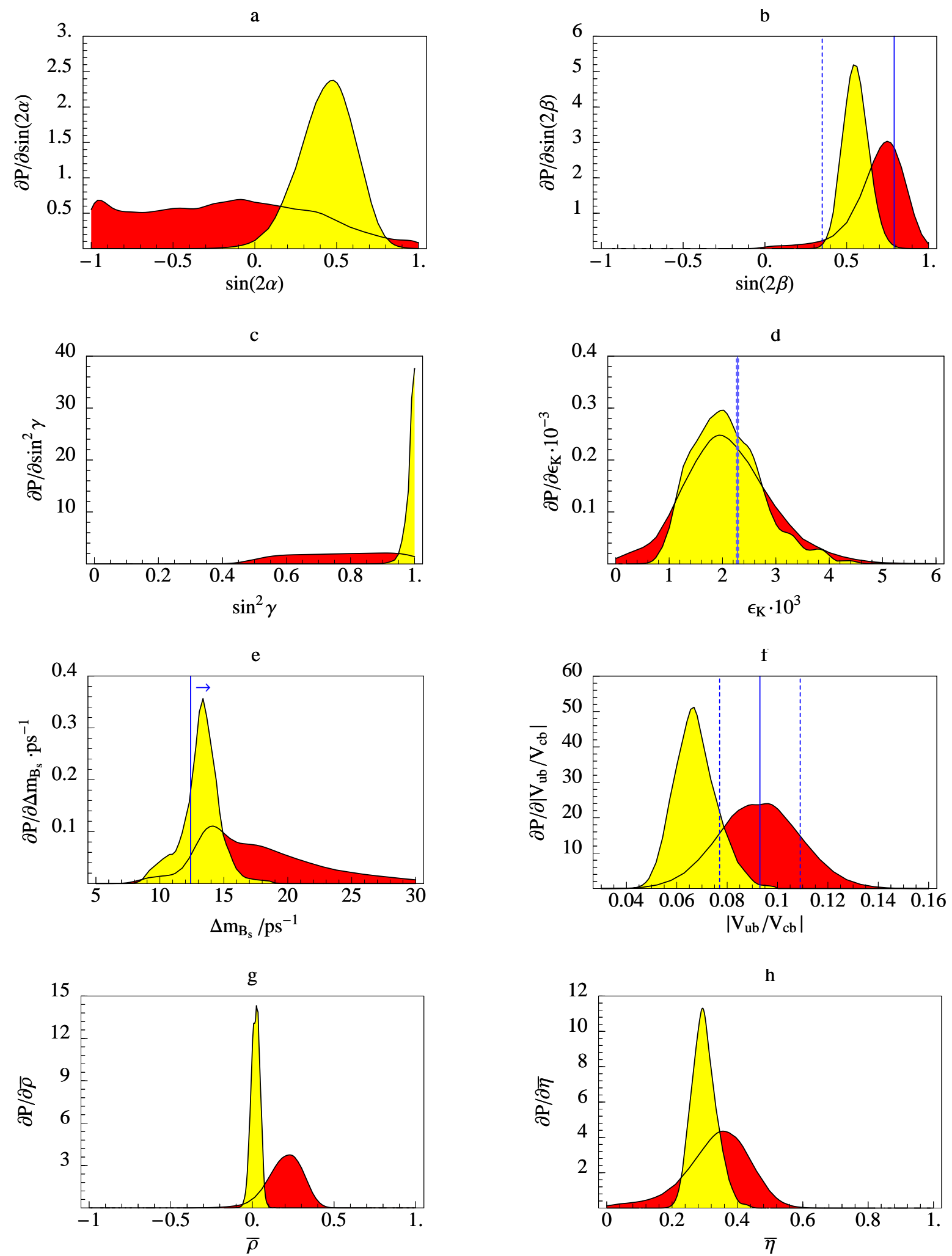

Figure 2: Probability distributions (lighter area: predictions; darker area: from the SM fit, excluding $\epsilon_{K}$ but taking $\eta>0$ ) for different observables: a) $\sin 2 \alpha$, b) $\sin 2 \beta$, c) $\sin ^{2} \gamma$, d) $\epsilon_{K}$, e) $\Delta m_{B_{s}}$, f) $\left|V_{u b} / V_{c b}\right|$, g) $\rho$, h) $\eta$. 

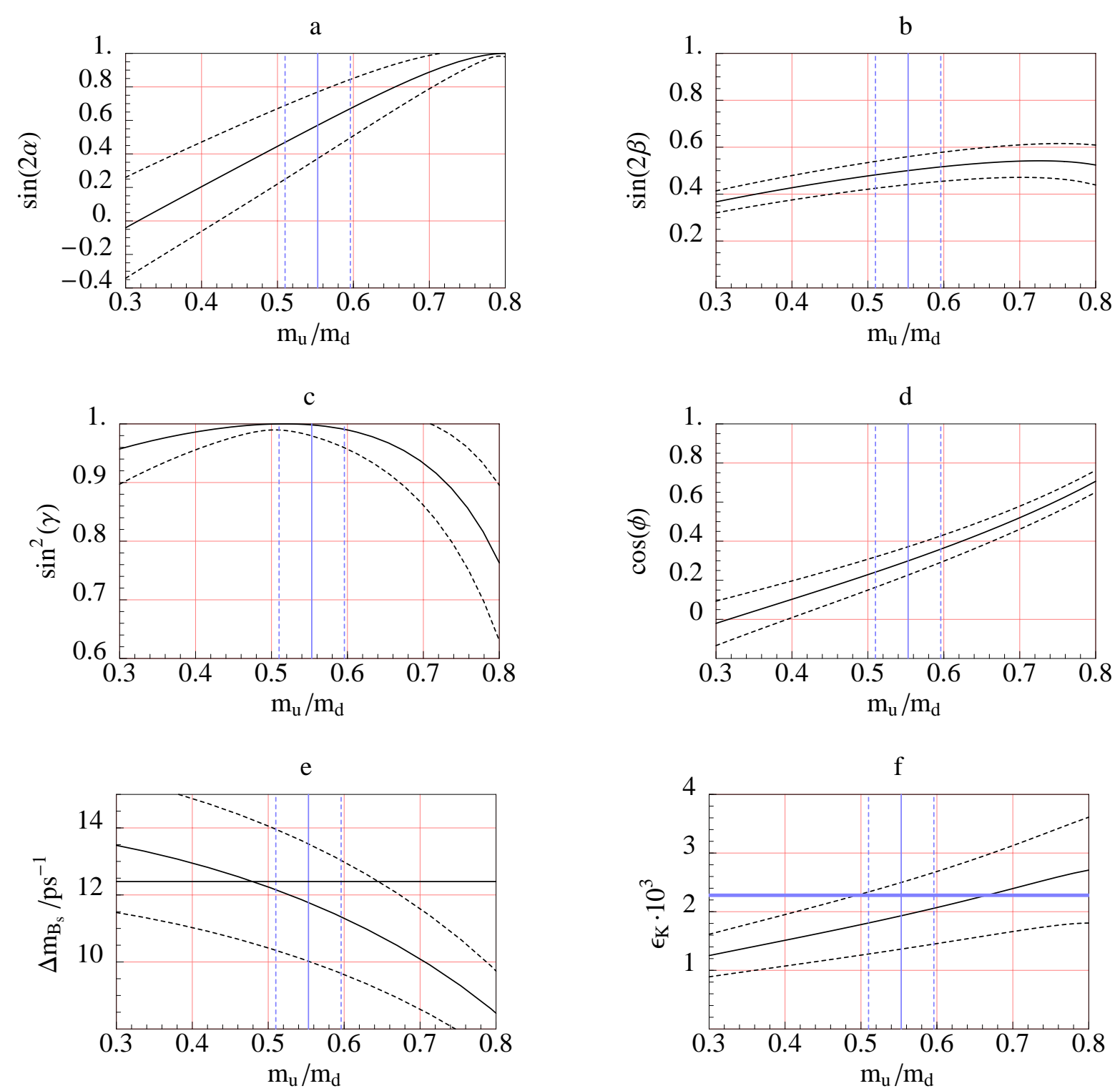

Figure 3: Predictions as functions of $m_{u} / m_{d}$ for different observables: a) $\sin 2 \alpha$, b) $\sin 2 \beta$, c) $\sin ^{2} \gamma$, d) $\cos \phi$, e) $\Delta m_{B_{s}}$, f) $\epsilon_{K}$. 